\title{
DERIVATIONS ON ALGEBRAS OF UNBOUNDED OPERATORS \\ BY
}

\author{
ATSUSHI INOUE AND SCHÔICHI ÔTA
}

\begin{abstract}
This paper is a study of derivations on unbounded operator algebras in connection with those in operator algebras.

In particular we study spatiality of derivations in several situations. We give the characterization of derivations on general *-algebras by using positive linear functionals. We also show that a derivation with some range-property on a left $E W^{\#}$-algebra induced by an unbounded Hilbert algebra is strongly implemented by an operator which belongs to an algebra of measurable operators.
\end{abstract}

1. Introduction. Derivations in operator algebras $\left(C^{*}\right.$-algebras and von Neumann algebras) have been investigated by many authors and many useful results have been obtained. In particular, it is well known [18] that every bounded derivation on a von Neumann algebra is spatial; that is, it is implemented by an element of its von Neumann algebra. The structure of unbounded derivations in operator algebras is more complicated than that of bounded derivations. It is well known that a generator of a strongly continuous one-parameter subgroup of *-automorphisms of a $C^{*}$-algebra is a *-derivation which is implemented by a symmetric operator by giving some representation of its $C^{*}$-algebra on a Hilbert space. It seems to be important to investigate whether an unbounded closed derivation in a $C^{*}$-algebra is spatial by some faithful representation in a Hilbert space, or not [5].

Let $\delta$ be a *-derivation in a von Neumann algebra $\mathfrak{M}$ acting on a Hilbert space $\mathfrak{Q}$. Suppose that $\delta$ is implemented by a selfadjoint operator $H$ (possibly unbounded) in $\mathfrak{Q}$ with $H \mathscr{D}(H) \subset \mathscr{D}(H)$, where $\mathscr{D}(H)$ denotes the domain of $H$. Then we can easily construct a derivation on an unbounded operator algebra generated by the domain $\mathscr{D}(\delta)$ of $\delta$ and $H$. Furthermore, if a derivation $\delta$ on a particular unbounded operator algebra (an $E W^{\#}$-algebra) $\mathcal{Q}$ is implemented by a selfadjoint element $H$ in $Q$, then we can also construct a $\sigma$-strongly closed *-derivation in a von Neumann algebra.

In this point of view, we shall study in $\$ 4$ the spatiality of derivations on unbounded operator algebras.

In $\$ 3$ we shall consider derivations on *-algebras with identity and extend a derivation on *-algebras with identity to one on unbounded operator algebras by using the G-N-S representation induced by some positive linear functional, and we characterize the spatiality of its extended derivation by a positive linear functional.

Received by the editors May 24, 1979.

AMS (MOS) subject classifications (1970). Primary 46K10, 46K15, 46L20; Secondary 46H15, 46H25, 46L05, 46L10.

Key words and phrases. Unbounded operator algebra, derivation. 
2. Preliminaries. We start with some notations and definitions of unbounded operator algebras. Let $\mathscr{D}$ be a pre-Hilbert space with the Hilbert space $\mathscr{E}$ which is the completion of $\mathscr{D}$. By $\mathcal{L}(\mathscr{D})$ we denote the algebra of all linear operators on $\mathscr{D}$. By $\mathcal{L}^{\#}(\mathscr{D})$ we denote the set of all operators $A \in \mathcal{L}(\mathscr{D})$ for which there exists an operator $A^{\#} \in \mathcal{L}(\mathscr{D})$ such that

$$
(A \xi \mid \eta)=\left(\xi \mid A^{\#} \eta\right)
$$

for every $\xi, \eta \in \mathscr{D}$. It is easily seen that each operator in $\mathcal{L}^{\#}(\mathscr{D})$ is closable in $\mathscr{Q}$ and $\mathcal{L}^{\#}(\mathscr{D})$ is an involutive algebra with the involution $A \rightarrow A^{\#}$. An involutive subalgebra of $\mathfrak{L}^{\#}(\mathscr{D})$ is called a \#-algebra on $\mathscr{D}$. We note that a \#-algebra with identity operator is said to be an $\vartheta_{p}^{*}$-algebra on $\mathscr{D}$ by $\mathrm{G}$. Lassner.

Let $Q$ be a \#-algebra on $\mathscr{D}$. We denote by $\mathbb{Q}_{b}$ the bounded part of $\mathcal{Q}$, that is, $\mathbb{Q}_{b}=\{A \in \mathbb{Q} ; \bar{A} \in \mathscr{B}(\mathfrak{E})\}$, where $\bar{A}$ denotes the smallest closed extension of $A$ and $\mathscr{B}(\mathfrak{Q})$ denotes the algebra of all bounded linear operators on $\mathfrak{Q}$. A \#-algebra $\mathbb{Q}$ on $\mathscr{D}$ with the identity operator $I$ is called a symmetric \#-algebra on $\mathscr{D}$ if $\left(I+A^{\#} A\right)^{-1}$ exists and lies in $\mathbb{Q}_{b}$ for all $A \in \mathbb{Q}$. If $\mathbb{Q}$ is a symmetric \#-algebra on $\mathscr{Q}$ and $\overline{\mathbb{Q}_{b}}$ is a $C^{*}$-algebra (resp. $W^{*}$-algebra), then $\mathcal{Q}$ is called an $E C^{\# \text {-algebra on }}$ $\mathscr{D}$ over $\overline{\mathbb{Q}_{b}}$ (resp. an $E W^{\#}$-algebra on $\mathscr{D}$ over $\overline{Q_{b}}$ ), where $\overline{\mathbb{Q}_{b}}$ is the set of all $\bar{A}$ with $A$ in $Q_{b}$. For a more complete discussion of \#-algebras the reader is referred to [1], [8]-[11], [13].

Let $\mathscr{B}$ be an involutive algebra (*-algebra) with involution $A \rightarrow A^{*}$. By a *-derivation in $\mathscr{B}$ we mean a linear mapping $\delta$ of the domain $\mathscr{D}(\delta)$, which is a *-subalgebra of $\mathscr{B}$, into $\mathscr{B}$ satisfying that for each $A, B \in \mathscr{B}$

$$
\delta(A B)=\delta(A) B+A \delta(B), \quad \delta\left(A^{*}\right)=\delta(A)^{*} .
$$

In particular a $*$-derivation in a \#-algebra $Q$ with $\mathscr{D}(\delta)=\mathscr{Q}$ is called a \#-derivation on $\mathbb{Q}$. If a \#-derivation on a \#-algebra $\mathbb{Q}$ is continuous with respect to the topology $\tau$ on $Q, \delta$ is said to be $\tau$-continuous. We set $\mathbb{Q}_{0}=\left\{A \in \mathbb{Q}_{b} ; \delta(A) \in \mathbb{Q}_{b}\right\}$. It is easily seen that $\overline{Q_{0}}$ is a *-subalgebra of a *-algebra $\overline{Q_{b}}$. Hence we define a *-derivation in $\overline{Q_{b}}$ with the domain $\overline{Q_{0}}$ as follows

$$
\delta_{0}(\bar{A})=\overline{\delta(A)} \quad\left(A \in \mathbb{Q}_{0}\right) .
$$

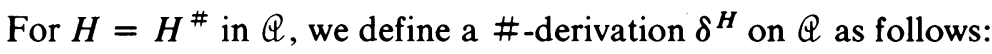

$$
\delta^{H}(A)=i[H, A] \quad(=i(H A-A H))
$$

for $A \in \mathbb{Q}$. Such a derivation is called a spatial derivation implemented by $H$.

Proposition 2.1. Let $Q$ be an $E W^{\#}$-algebra on $\mathscr{D}$ and let $\delta^{H}$ be a spatial derivation on $\mathcal{Q}$. Then $\left(\delta^{H}\right)_{0}$ is a $\sigma$-weakly closed *-derivation in the von Neumann algebra $\overline{Q_{b}}$ with the $\sigma$-weakly dense domain $\overline{Q_{0}}$.

Proof. We first show that $\overline{\mathbb{Q}_{0}}$ is $\sigma$-weakly dense in $\overline{\mathbb{Q}_{b}}$. Since $\mathcal{Q}$ is an $E W^{\#}$-algebra, $\bar{H}$ is a selfadjoint operator with the spectral resolution $\bar{H}=\int_{-\infty}^{\infty} d E(\lambda)$. For each $A \in \mathbb{Q}_{b}$ we put $A_{n}=E\left(\lambda_{n}\right) A E\left(\lambda_{n}\right)$, where $\left\{\lambda_{n}\right\}$ is an increasing sequence with $\lim _{n \rightarrow \infty} \lambda_{n}=\infty$ and $E\left(\lambda_{n}\right)$ converges $\sigma$-strongly to the identity operator $I$. Then we have $\delta^{H}\left(A_{n}\right)=i\left[H, A_{n}\right]=i\left\{H E\left(\lambda_{n}\right) A E\left(\lambda_{n}\right)-E\left(\lambda_{n}\right) A E\left(\lambda_{n}\right) H\right\} \in \mathbb{Q}_{b}$, which implies $A_{n} \in \mathbb{Q}_{0}$. Since $\bar{A}_{n}=E\left(\lambda_{n}\right) \bar{A} E\left(\lambda_{n}\right)$ converges $\sigma$-weakly to $\bar{A}$, $\mathbb{Q}_{0}$ is $\sigma$-weakly 
dense in $\overline{\mathbb{Q}_{b}}$. Now we recall the $(\mathscr{Q})$ - $\sigma$-weak topology simply denoted by $t_{\sigma-w}^{\mathfrak{Q}}$. Let $\mathfrak{Q}_{\infty}$ be the Hilbert direct sum of the Hilbert spaces $\mathfrak{Q}_{n}$ with $\mathfrak{Q}_{n}=\mathfrak{G}(n=1,2, \ldots)$. We set

$$
\begin{aligned}
& \mathscr{D}_{\infty}(\mathbb{Q})=\left\{\left\{\xi_{n}\right\} \in \mathfrak{Q}_{\infty} ; \xi_{n} \in \mathscr{D}(n=1,2, \ldots)\right. \text { and } \\
&\left.\sum_{n=1}^{\infty}\left\|A \xi_{n}\right\|^{2}<\infty \text { for all } A \in \mathbb{Q}\right\}
\end{aligned}
$$

and

$$
P_{\left\{\xi_{n}\right\},\left\{\eta_{n}\right\}}(A)=\left|\sum_{n=1}^{\infty}\left(A \xi_{n} \mid \eta_{n}\right)\right|
$$

where $A \in \mathbb{Q},\left\{\xi_{n}\right\},\left\{\eta_{n}\right\} \in \mathscr{D}_{\infty}(\mathbb{Q})$. It is easily seen that $\mathbb{Q}$ is a locally convex *-algebra equipped with the locally convex topology $t_{\sigma-w}^{\mathcal{Q}}$ generated by the family $\left\{P_{\left\{\xi_{n}\right\},\left\{\eta_{n}\right\}}(\cdot) ;\left\{\xi_{n}\right\},\left\{\eta_{n}\right\} \in \mathscr{D}_{\infty}(\mathbb{Q})\right\}$. The derivation $\delta^{H}$ is $t_{\sigma-w}^{\mathbb{Q}}$-continuous on $\mathbb{Q}$ and so $\left(\delta^{H}\right)_{0}$ is $\sigma$-weakly closed in $\overline{Q_{b}}$. This completes the proof of Proposition 2.1.

EXAmPle 2.2 (DIRECT SUMS OF \#-DERIVATIONS). Let $\left\{\mathbb{Q}_{i}\right\}$ be a sequence of \#-algebras $\mathscr{Q}_{i}$ on $\mathscr{D}_{i}$ with the identity operators $I_{i}$. We denote by $\mathscr{D}$ the set $\Sigma^{\oplus} \mathscr{D}_{i}$ of all elements $\xi=\left(\xi_{i}\right)$, where $\xi_{i} \in \mathscr{D}_{i}$ and $\xi_{i}=0$ except for finitely many indices $i$ and also denote by $Q$ the Cartesian product $\prod_{i=1}^{\infty} Q_{i}$ of the \#-algebras $\mathbb{Q}_{i}$. Then $Q$ is a \#-algebra on $\mathscr{D}$ with identity $I=\left(I_{i}\right)$ by the usual operations. We note that for $A=\left(A_{i}\right) \in Q, A^{\#}=\left(A_{i}^{\#}\right)$. Then each \#-derivation $\delta$ on $Q$ is represented by the direct sum of \#-derivations $\delta_{i}$ on $\mathbb{Q}_{i}$, that is, $\delta(A)=\left(\delta_{i}\left(A_{i}\right)\right)$ for $A=\left(A_{i}\right) \in \mathbb{Q}$. In fact, for every $A_{i}$ in $\mathbb{Q}_{i}$ we set

$$
C=\left(\delta\left(A_{i}\right)_{1}^{\#}, \delta\left(A_{i}\right)_{2}^{\#}, \ldots, \delta\left(A_{i}\right)_{i-1}^{\#}, \stackrel{i}{\check{0}}, \delta\left(A_{i}\right)_{i+1}^{\#}, \delta\left(A_{i}\right)_{i+2}^{\#}, \ldots\right) \in \mathbb{Q} .
$$

Then we have

$$
\begin{aligned}
\delta\left(A_{i} C\right)= & \delta\left(A_{i}\right) C+A_{i} \delta(C) \\
= & \left(\delta\left(A_{i}\right)_{1} \delta\left(A_{i}\right)_{1}^{\#}, \delta\left(A_{i}\right)_{2} \delta\left(A_{i}\right)_{2}^{\#}, \ldots, \delta\left(A_{i}\right)_{i-1} \delta\left(A_{i}\right)_{i-1}^{\#},\right. \\
& \left.A_{i} \delta(C)_{i}, \delta\left(A_{i}\right)_{i+1} \delta\left(A_{i}\right)_{i+1}^{\#}, \delta\left(A_{i}\right)_{i+2} \delta\left(A_{i}\right)_{i+2}^{\#}, \ldots\right) .
\end{aligned}
$$

Since $A_{i} C=0$, we have $\delta\left(A_{i}\right)_{k} \delta\left(A_{i}\right)_{k}^{\#}=0$ for $k \neq i$ and so $\delta\left(A_{i}\right)_{k}=0$ for $k \neq i$. This implies that each $Q_{i}$ is invariant under $\delta$, and hence we can define a linear mapping $\delta_{i}$ on $Q_{i}$ as the restriction of $\delta$ to $Q_{i}$. It is easily proved that each $\delta_{i}$ is a \#-derivation on $\mathbb{Q}_{i}$ and $\delta(A)_{i}=\delta_{i}\left(A_{i}\right)$ for $A=\left(A_{i}\right) \in \mathbb{Q}$. Suppose that in the above situation, each $\mathcal{Q}_{i}$ is a von Neumann algebra on a Hilbert space $\mathfrak{S}_{i}$ $\left(\mathfrak{S}_{i}=\mathscr{D}_{i}\right)$. Then $Q=\prod_{i=1}^{\infty} Q_{i}$ is an $E W^{\#}$-algebra on $\mathscr{D}=\Sigma_{i}^{\oplus} \mathfrak{S}_{i}$ and every \#-derivation on $\mathcal{Q}$ is a spatial derivation on $\mathbb{Q}$. This follows from the well-known fact [18] that every bounded *-derivation $\delta$ on a von Neumann algebra $\mathfrak{M}$ is represented as $\delta(A)=i[H, A]$ for $A \in \mathfrak{M}$, where $H$ is a selfadjoint element in $\mathfrak{M}$.

3. Derivations on *-algebras. Let $\mathbf{A}$ be a *-algebra with identity $e$ and let $f$ be a positive linear functional on $\mathbf{A}$, that is, $f\left(x^{*} x\right) \geqslant 0$ for every $x \in \mathbf{A}$. We consider the G-N-S representation induced by $f$. The elements $a$ in $\mathbf{A}$ with $f\left(a^{*} a\right)=0$ form a left ideal $\mathfrak{R}_{f}$ in $\mathbf{A}$. For $a$ in $\mathbf{A}$ we denote by $\lambda_{f}(a)$ the coset of $\mathbf{A} / \mathscr{N}_{f}\left(\equiv \lambda_{f}(\mathbf{A})\right)$ 
which contains $a$ and we define an inner product by $\left(\lambda_{f}(a) \mid \lambda_{f}(b)\right)=f\left(b^{*} a\right)$. Then $\lambda_{f}(\mathbf{A})$ becomes a pre-Hilbert space with the completion $\mathfrak{B}_{f}$. For each $a \in \mathbf{A}$, we define a linear operator on $\lambda_{f}(\mathbf{A})$ by $\pi_{f}(a) \lambda_{f}(b)=\lambda_{f}(a b)(b \in \mathbf{A})$. It is easily seen that $\pi_{f}(a)$ is closable in $\mathfrak{Q}_{f}$ and for $a, b \in \mathbf{A}$ we have $\pi_{f}(\alpha a+\beta b)=\alpha \pi_{f}(a)+$ $\beta \pi_{f}(b)$ and $\pi_{f}(a) \pi_{f}(b)=\pi_{f}(a b)(\alpha, \beta \in \mathbf{C})$. Then $\pi_{f}(\mathbf{A})$ is a \# -algebra on $\lambda_{f}(\mathbf{A})$ with $\pi_{f}(a)^{\#}=\pi_{f}\left(a^{*}\right)$. Now let $\pi$ be an involution-preserving homomorphism of a *-algebra $\mathbf{A}$ into a \#-algebra $Q$ and let $\delta$ be a *-derivation on $\mathbf{A}$. In the rest of this paper, we only consider everywhere defined derivations. If $\delta$ preserves the kernel of $\pi$, i.e., $\delta(\operatorname{ker} \pi) \subset \operatorname{ker} \pi$, we can define a \#-derivation $\delta_{\pi}$ induced by $\delta$ on a \#-algebra $\pi(\mathbf{A})$ by defining $\delta_{\pi}(\pi(a))=\pi(\delta(a))$ for $a \in \mathbf{A}$.

The following proposition is well known in the case of unbounded derivations in $C^{*}$-algebras [2, Theorem 4] and is proved in the same way.

Proposition 3.1. Let $\mathbf{A}$ be a *-algebra with identity $e$ and let $\delta$ be a $a$-derivation on A. Suppose that there exists a positive linear functional $f$ with $f(\delta(a))=0$ for $a \in \mathbf{A}$. Then there exists an element $H=H^{\#}$ of $\mathcal{L}^{\#}\left(\lambda_{\gamma}(\mathbf{A})\right)$ such that

$$
\delta_{\pi_{f}}\left(\pi_{f}(a)\right)=i\left[H, \pi_{f}(a)\right] \text { for } a \in \mathbf{A} \text {. }
$$

Using the above proposition, we can prove the following proposition in the same way as in [2, Theorem 8].

PROPOSITION 3.2. If $\mathbb{Q}$ is a \#-algebra on $\mathscr{D}$ containing all finite rank operators in $\mathfrak{L}^{\#}(\mathscr{D})$ and $\delta$ is a \#-derivation on $\mathcal{Q}$, then there exists an element $H=H^{\#}$ of $\mathfrak{L}^{\#}(\mathcal{D})$ such that

$$
\delta(A)=i[H, A] \text { for all } A \in \mathbb{Q} .
$$

Chernoff proved in [7] that if $X$ is a normed space and $\delta$ is a derivation on the algebra of all linear continuous operators, then $\delta$ is spatial. In the case of derivations on unbounded operator algebras, we have

Corollary 3.3. Each \#-derivation on $\mathfrak{L}^{\#}(\mathcal{D})$ is a spatial derivation implemented by $H=H^{\#}$ in $\mathcal{L}^{\#}(\mathscr{D})$.

Let $f$ be a positive linear functional on a *-algebra $\mathbf{A}$ with identity $e$. We set

$$
\mathscr{D}\left(\pi_{f}^{*}\right)=\bigcap_{a \in \mathbf{A}} \mathscr{D}\left(\pi_{f}(a)^{*}\right)
$$

and $\pi_{f}^{*}(a) \xi=\pi_{f}\left(a^{*}\right)^{*} \xi\left(a \in \mathbf{A}, \xi \in \mathscr{D}\left(\pi_{f}^{*}\right)\right)$. It follows from [15, Lemma 4.1] that $\pi_{f}^{*}$ is a homomorphism of $\mathbf{A}$ into $\mathcal{L}\left(\mathscr{D}\left(\pi_{f}^{*}\right)\right)$. We remark that $\mathscr{Q}\left(\pi_{f}^{*}\right) \supset \lambda_{f}(\mathbf{A})$ and $\pi_{f}^{*}(a)$ coincides with $\pi_{f}(a)$ on $\lambda_{f}(\mathbf{A})$.

THEOREM 3.4. Let $\delta$ be a*-derivation on a *-algebra $\mathbf{A}$ with identity $e$ and let $f$ be a positive linear functional on $\mathbf{A}$. The following conditions are equivalent.

(1) $|f(\delta(b a))|^{2} \leqslant \gamma_{b} f\left(a^{*} a\right)$ for all $a, b \in \mathbf{A}$, where $\gamma_{b}$ is a constant depending only on $b$.

(2) There exists a linear operator $H$ in $\mathscr{L}\left(\lambda_{f}(\mathbf{A}), \mathscr{D}\left(\pi_{f}^{*}\right)\right)$ satisfying the following conditions $(\mathrm{a}) \sim$ (c): 
(a) $H$ is a symmetric operator on $\mathfrak{G}_{f}$.

(b) $\left(H \lambda_{f}(e) \mid \lambda_{f}(a)\right)=-\left(\lambda_{f}\left(a^{*}\right) \mid H \lambda_{f}(e)\right)$ for all $a \in \mathbf{A}$.

(c) $\delta_{\pi_{f}}\left(\pi_{f}(a)\right) \lambda_{f}(b)=i\left[H, \pi_{f}^{*}(a)\right] \lambda_{f}(b)$ for all $a, b \in \mathbf{A}$, where $\mathcal{L}\left(\lambda_{f}(\mathbf{A}), \mathscr{D}\left(\pi_{f}^{*}\right)\right)$ denotes the set of all linear transformations of $\lambda_{f}(\mathbf{A})$ into $\mathscr{D}\left(\pi_{f}^{*}\right)$.

Proof. $(1) \Rightarrow(2)$. The idea of this proof is based on [4]. We define a linear functional $\phi$ on $\lambda_{f}(\mathrm{~A})$ by $\phi\left(\lambda_{f}(a)\right)=i f(\delta(a))$. From the condition (1) we have $\left|\phi\left(\lambda_{f}(a)\right)\right| \leqslant \gamma_{e}^{1 / 2}\left\|\lambda_{f}(a)\right\|$ and so there exists a vector $\xi$ in $\mathfrak{B}_{f}$, by the Riesz representation theorem, such that if( $\delta(a))=\left(\lambda_{f}(a) \mid \xi\right)$ for all $a \in \mathbf{A}$. Furthermore we have

$$
\left|\left(\pi_{f}(b) \lambda_{f}(a) \mid \xi\right)\right|=|i f(\delta(b a))| \leqslant \gamma_{b}^{1 / 2}\left\|\lambda_{f}(a)\right\|
$$

for all $a, b \in \mathbf{A}$, which implies that $\xi$ belongs to $\mathscr{D}\left(\pi_{f}^{*}\right)$. Now we define a linear operator $H$ in $\mathfrak{S}_{f}$ by

$$
H \lambda_{f}(a)=-i \lambda_{f}(\delta(a))-\frac{1}{2} \pi_{f}^{*}(a) \xi
$$

We show that $H$ has the desired properties. Since

$$
\left(\lambda_{f}\left(a^{*}\right) \mid \xi\right)=i f\left(\delta\left(a^{*}\right)\right)=\overline{-i f(\delta(a))}=-\left(\xi \mid \lambda_{f}(a)\right)
$$

for all $a \in \mathbf{A}$, we have

$$
\begin{aligned}
\left(H \lambda_{f}(a) \mid \lambda_{f}(b)\right)-\left(\lambda_{f}(a) \mid\right. & \left.H \lambda_{f}(b)\right)=\left(-i \lambda_{f}(\delta(a)) \mid \lambda_{f}(b)\right)-\frac{1}{2}\left(\pi_{f}^{*}(a) \xi \mid \lambda_{f}(b)\right) \\
& -\left(\lambda_{f}(a) \mid-i \lambda_{f}(\delta(b))\right)+\frac{1}{2}\left(\lambda_{f}(a) \mid \pi_{f}^{*}(b) \xi\right) \\
= & -i f\left(b^{*} \delta(a)\right)-\frac{1}{2}\left(\xi \mid \lambda_{f}\left(a^{*} b\right)\right)-i f\left(\delta(b)^{*} a\right)+\frac{1}{2}\left(\lambda_{f}\left(b^{*} a\right) \mid \xi\right) \\
= & -i f\left(\delta\left(b^{*} a\right)\right)+\left(\lambda_{f}\left(b^{*} a\right) \mid \xi\right)=0
\end{aligned}
$$

for all $a, b \in \mathbf{A}$. Hence $H$ is a well-defined symmetric operator and belongs to $\mathcal{L}\left(\lambda_{f}(\mathrm{~A}), \mathscr{D}\left(\pi_{f}^{*}\right)\right)$ by the definition. From the equality that $H \lambda_{f}(e)=-\frac{1}{2} \xi$, we find the condition (b). Finally using the homomorphism of $\pi_{f}^{*}$, for $a, b \in \mathbf{A}$ we have

$$
\begin{aligned}
i\left[H, \pi_{f}^{*}(a)\right] \lambda_{f}(b) & =i\left\{H \pi_{f}(a) \lambda_{f}(b)-\pi_{f}^{*}(a) H \lambda_{f}(b)\right\} \\
& =i\left\{H \lambda_{f}(a b)-\pi_{f}^{*}(a) H \lambda_{f}(b)\right\} \\
& =i\left\{-i \lambda_{f}(\delta(a b))-\frac{1}{2} \pi_{f}^{*}(a b) \xi-\pi_{f}^{*}(a)\left(-i \lambda_{f}(\delta(b))-\frac{1}{2} \pi_{f}^{*}(b) \xi\right)\right\} \\
& =\lambda_{f}(\delta(a b))-\frac{1}{2} i \pi_{f}^{*}(a b) \xi-\lambda_{f}(a \delta(b))+\frac{1}{2} i \pi_{f}^{*}(a b) \xi \\
& =\lambda_{f}(\delta(a) b)=\delta_{\pi_{f}}\left(\pi_{f}(a)\right) \lambda_{f}(b) .
\end{aligned}
$$

This implies $(1) \Rightarrow(2)$.

(2) $\Rightarrow$ (1). For $a, b \in \mathbf{A}$, we have

$$
\begin{aligned}
|f(\delta(b a))| & \leqslant|f(\delta(b) a)|+|f(b \delta(a))| \\
& \leqslant f\left(\delta(b) \delta(b)^{*}\right)^{1 / 2} f\left(a^{*} a\right)^{1 / 2}+\left|\left(\lambda_{f}(\delta(a)) \mid \lambda_{f}\left(b^{*}\right)\right)\right|
\end{aligned}
$$


and

$$
\begin{aligned}
\left|\left(\lambda_{f}(\delta(a)) \mid \lambda_{f}\left(b^{*}\right)\right)\right| & =\left|i\left(H \pi_{f}^{*}(a) \lambda_{f}(e)-\pi_{f}^{*}(a) H \lambda_{f}(e) \mid \lambda_{f}\left(b^{*}\right)\right)\right| \\
& \leqslant\left|\left(H \lambda_{f}(a) \mid \lambda_{f}\left(b^{*}\right)\right)\right|+\left|\left(H \lambda_{f}(e) \mid \pi_{f}\left(a^{*}\right) \lambda_{f}\left(b^{*}\right)\right)\right| \\
& =\left|\left(\lambda_{f}(a) \mid H \lambda_{f}\left(b^{*}\right)\right)\right|+\left|\left(\lambda_{f}(b a) \mid H \lambda_{f}(e)\right)\right| \quad \text { (condition (b)) } \\
& \leqslant\left\|H \lambda_{f}\left(b^{*}\right)\right\| f\left(a^{*} a\right)^{1 / 2}+\left|\left(\lambda_{f}(a) \mid \pi_{f}(b)^{*} H \lambda_{f}(e)\right)\right| \\
& \leqslant\left(\left\|H \lambda_{f}\left(b^{*}\right)\right\|+\left\|\pi_{f}(b)^{*} H \lambda_{f}(e)\right\|\right) f\left(a^{*} a\right)^{1 / 2}
\end{aligned}
$$

Therefore there exists a constant $\gamma_{b}$ depending only on $b$ such that $|f(\delta(b a))|^{2} \leqslant$ $\gamma_{b} f\left(a^{*} a\right)$ for each $a, b \in \mathbf{A}$. This completes the proof of Theorem 3.4.

4. Derivations on left $E W^{\#}$-algebras. In this section we consider derivations in a particular \#-algebra (a left $E W^{\#}$-algebra) which has an interesting structure in \#-algebras. We recall some of the definitions and results concerning unbounded Hilbert algebras and left $E W^{\#}$-algebras and refer to [9], [10] for further details.

Let $\mathfrak{U}$ be a pre-Hilbert space with inner product $(\cdot \mid \cdot)$ and be a $*$-algebra. Let $\mathfrak{S}(\mathfrak{A})$ be the completion of $\mathfrak{A}$. Suppose $\mathfrak{A}$ satisfies the following conditions:

(1) $(\xi \mid \eta)=\left(\eta^{*} \mid \xi^{*}\right)$

(2) $(\xi \eta \mid \zeta)=\left(\eta \mid \xi^{*} \zeta\right)$

for every $\xi, \eta$ and $\zeta \in \mathfrak{A}$. For each $\xi \in \mathfrak{A}$, we define $\pi(\xi)$ and $\pi^{\prime}(\xi)$ by $\pi(\xi) \eta=\xi \eta$ and $\pi^{\prime}(\xi) \eta=\eta \xi$ for $\eta \in \mathfrak{A}$. Then by the equalities (1) and (2) we see that both $\pi(\xi)$ and $\pi^{\prime}(\xi)$ are closable operators in $\mathfrak{Q}(\mathfrak{U})$ with domains $\mathfrak{U}$ satisfying $\pi(\xi)^{*} \supset \overline{\pi\left(\xi^{*}\right)}$ and $\pi^{\prime}(\xi)^{*} \supset \overline{\pi^{\prime}\left(\xi^{*}\right)}$. Furthermore if $\mathfrak{A}$ satisfies the conditions (1), (2) and

(3) $\mathfrak{A}_{0}^{2}$ is dense in $\mathfrak{g}(\mathfrak{U})$,

where $\mathfrak{A}_{0}$ denotes the set of all elements $\xi$ of $\mathfrak{U}$ such that $\overline{\pi(\xi)}$ is a bounded operator on $\mathfrak{E}(\mathfrak{A})$, then $\mathfrak{A}$ is called an unbounded Hilbert algebra over $\mathfrak{A}_{0}$ in $\mathfrak{Q}(\mathfrak{A})$ and $\pi$ (resp. $\left.\pi^{\prime}\right)$ is called the left (resp. right) regular representation of $\mathfrak{A}$. If $\mathfrak{A}$ is an unbounded Hilbert algebra over $\mathfrak{A}_{0}$ in $\mathfrak{E}(\mathfrak{A})$, then we see that $\mathfrak{A}_{0}$ is a Hilbert algebra and the completion $\mathfrak{S}\left(\mathfrak{A}_{0}\right)$ of $\mathfrak{A}_{0}$ is the Hilbert space $\mathfrak{Q}(\mathfrak{U})(\equiv \mathfrak{Q})$. The involution * on $\mathfrak{A}$ is extended to an involution on $\mathfrak{G}$, which is also denoted by *.

Let $\pi_{0}$ (resp. $\pi_{0}^{\prime}$ ) be the left (resp. right) regular representation of the Hilbert algebra $\mathfrak{A}_{0}$. For each $x$ in $\mathfrak{S}$, we define linear operators $\pi_{0}(x)$ and $\pi_{0}^{\prime}(x)$ in $\mathfrak{G}$ by

$$
\pi_{0}(x) \xi=\overline{\pi_{0}^{\prime}(\xi)} x \quad \text { and } \quad \pi_{0}^{\prime}(x) \xi=\overline{\pi_{0}(\xi)} x
$$

for $\xi \in \mathfrak{A}_{0}$. Then both $\pi_{0}(x)$ and $\pi_{0}^{\prime}(x)$ are closable operators in $\mathfrak{Q}$ with domains $\mathfrak{A}_{0}$ such that

$$
\pi_{0}(x)^{*}=\overline{\pi_{0}\left(x^{*}\right)} \text { and } \pi_{0}^{\prime}(x)^{*}=\overline{\pi_{0}^{\prime}\left(x^{*}\right)} .
$$

Let $\mathscr{U}_{0}\left(\mathfrak{A}_{0}\right)$ (resp. $\left.\mathcal{V}_{0}\left(\mathfrak{A}_{0}\right)\right)$ be the left (resp. right) von Neumann algebra of the Hilbert algebra $\mathfrak{A}_{0}$ and let $\phi_{0}$ be the natural trace on $\mathscr{U}_{0}\left(\mathfrak{A}_{0}\right)^{+}$. We set $\left(\mathfrak{A}_{0}\right)_{b}=\{x$ $\left.\in \mathfrak{S} ; \overline{\pi_{0}(x)} \in \mathscr{B}(\mathfrak{L})\right\}$. Then $\left(\mathfrak{U}_{0}\right)_{b}$ is a Hilbert algebra containing $\mathfrak{A}_{0}$.

We next define the $L_{2}^{\omega}$-spaces with respect to the natural trace $\phi_{0}$ and the Hilbert algebra $\mathfrak{U}_{0}$ according to the noncommutative integration of Segal [21]. We denote by $L^{p}\left(\phi_{0}\right)=L^{p}(1 \leqslant p<+\infty)$ the $L^{p}$-space with respect to $\phi_{0}$ equipped with the 
$L^{p}$-norm $\|\cdot\|_{p}$ and also by $L^{\infty}$ the left von Neumann algebra $\mathscr{U}_{0}\left(\mathfrak{A}_{0}\right)$ with operator-norm $\|\cdot\|_{\infty}$.

Now we put $L_{2}^{\omega}\left(\phi_{0}\right)=\bigcap_{2 \leqslant p<\infty} L^{p}$ and

$$
L_{2}^{\omega}\left(\mathfrak{A}_{0}\right)=\left\{x \in \mathfrak{Q} ; \overline{\pi_{0}(x)} \in L_{2}^{\omega}\left(\phi_{0}\right)\right\} .
$$

Then $L_{2}^{\omega}\left(\mathfrak{A}_{0}\right)$ becomes an unbounded Hilbert algebra with the multiplication defined by $\xi \cdot \eta=\overline{\pi_{0}(\xi)} \eta\left(\xi, \eta \in L_{2}^{\omega}\left(\mathfrak{A}_{0}\right)\right)$ and involution *. Thus if $\mathfrak{A}$ is an unbounded Hilbert algebra over $\mathfrak{A}_{0}$ in $\mathfrak{E}$, then $\mathfrak{A}$ is an involutive subalgebra of $L_{2}^{\omega}\left(\mathfrak{A}_{0}\right)$.

Let $\pi_{2}^{\omega}$ be the left regular representation of $L_{2}^{\omega}\left(\mathfrak{A}_{0}\right)$. Then $L_{2}^{\omega}\left(\mathfrak{A}_{0}\right)$ is invariant under $\pi_{2}^{\omega}(\mathfrak{A})$ and also under $\mathscr{U}_{0}\left(\mathfrak{A}_{0}\right)$. Hence both $\pi_{2}^{\omega}(\mathfrak{U})$ and the restriction $\mathcal{U}_{0}\left(\mathfrak{A}_{0}\right) / L_{2}^{\omega}\left(\mathfrak{A}_{0}\right)$ of $\mathcal{U}_{0}\left(\mathfrak{A}_{0}\right)$ on $L_{2}^{\omega}\left(\mathfrak{A}_{0}\right)$ are \#-algebras on $L_{2}^{\omega}\left(\mathfrak{A}_{0}\right)$ under $\pi_{2}^{\omega}(\xi)^{\#}=$ $\pi_{2}^{\omega}\left(\xi^{*}\right)$ for $\xi \in \mathfrak{A}$ and $\left(T / L_{2}^{\omega}\left(\mathfrak{U}_{0}\right)\right)^{\#}=T^{*} / L_{2}^{\omega}\left(\mathfrak{A}_{0}\right)$ for $T \in \mathscr{U}_{0}\left(\mathfrak{A}_{0}\right)$. We denote by थ( $(\mathfrak{A})$ the \#-algebra on $L_{2}^{\omega}\left(\mathfrak{A}_{0}\right)$ generated by $\pi_{2}^{\omega}(\mathfrak{A})$ and $\mathscr{U}_{0}\left(\mathfrak{A}_{0}\right) / L_{2}^{\omega}\left(\mathfrak{A}_{0}\right)$. We see that $\mathcal{U}(\mathfrak{A})$ is an $E W^{\#}$-algebra over $\mathscr{U}_{0}\left(\mathfrak{U}_{0}\right)$ on $L_{2}^{\omega}\left(\mathfrak{A}_{0}\right)$, which is called a left $E W^{\#}$-algebra of $\mathfrak{A}$. We remark that each element in $\overline{\mathscr{U}(\mathfrak{A})}$ is represented by the sum of an element in $L^{\infty}$ and an element in $L^{p}$ with any fixed $p(2 \leqslant p<+\infty)$.

Let $\Re$ be the set of all measurable operators with respect to $\phi_{0}$. It is well known [8] that $\mathscr{N}$ is a *-algebra under the operations of strong product, strong sum and strong scalar multiplication, and the adjoint operation.

We now introduce the strong commutator in $\Re$ as follows:

$$
[A, B]=A \cdot B-B \cdot A \text { for all } A, B \in \mathfrak{T} \text {. }
$$

LEMMA 4.1. Let $\mathfrak{A}$ be an unbounded Hilbert algebra over $\mathfrak{A}_{0}$ in $\mathfrak{G}$. Let $\mathbb{Q}$ be the left $E W^{\#}$-algebra $\mathcal{Q}(\mathfrak{A})$ induced by $\mathfrak{A}$ and let $\mathbb{Q}_{u}$ be the set of all unitary elements of $\mathbb{Q}$. If $\delta$ is $a$ \#-derivation on $Q$ with the range in $\pi_{2}^{\omega}\left(L_{2}^{\omega}\left(\mathfrak{A}_{0}\right)\right)$, then for each $p$ $(2 \leqslant p<\infty)$ there exists a selfadjoint operator $H_{p}$ in $L^{p}$ such that $\overline{\delta(A)}=i \cdot\left[H_{p}, \bar{A}\right]$ for all $A$ in $\mathbb{Q}_{b}$.

Proof. We shall show this lemma by the application of the Ryll-Nardzewski's fixed point theorem [14], [17]. We note that $\bar{Q}$ is contained in $L^{p}(2 \leqslant p<\infty)$. We denote by $\mathscr{K}$ the set of all $U^{\#} \delta(U)$ with $U \in \mathbb{Q}_{u}$ and by 2 the $\sigma\left(L^{p},\left(L^{p}\right)^{*}\right)$-closed convex hull of $\overline{\mathcal{K}}$. It is easily seen that $L^{p}$ is a Banach $\overline{\mathbb{Q}}_{b}$-module. We set $\delta_{b}(\bar{A})=\overline{\delta(A)}$ for $A \in \mathbb{Q}_{b}$. Then $\delta_{b}$ is a derivation of the $C^{*}$-algebra $\overline{\mathbb{Q}_{b}}$ into the Banach $\bar{Q}_{b}$-module $L^{p}$. It hence follows from [16, Theorem 2] that $\delta_{b}$ is continuous. This implies that 2 is a bounded subset of the Banach space $L^{p}$. Since $L^{p}$ is reflexive, it follows from the Alaoglu theorem that 2 is $\sigma\left(L^{p},\left(L^{p}\right)^{*}\right)$-compact. We now define an affine map on $L^{p}$ for each $U \in \mathbb{Q}_{u}$ as follows:

$$
A_{U}(S)=U^{*} S \cdot U+\overline{U^{\#} \delta(U)}
$$

for $S \in L^{p}$. Since $\bar{U} T \cdot U^{*} \in\left(L^{p}\right)^{*}$ for all $U \in \mathbb{Q}_{u}$ and $T \in\left(L^{p}\right)^{*}$, for each $U \in \mathbb{Q}_{u}$ the affine map $A_{U}$ is $\sigma\left(L^{p},\left(L^{p}\right)^{*}\right)$-continuous. Since for $U, V \in \mathbb{Q}_{u}$ we have

$$
\begin{aligned}
A_{V}\left(U^{*} \overline{\delta(U)}\right) & =V^{*} U^{*} \overline{\delta(U)} \cdot \bar{V}+V^{*} \overline{\delta(V)} \\
& =V^{*} U^{*}\{\overline{\delta(U)} \cdot \bar{V}+\bar{U} \overline{\delta(V)}\}=(U V)^{*} \overline{\delta(U V)}
\end{aligned}
$$


the affine map $A_{V}$ leaves 2 invariant. Since $A_{U} A_{V}(T)=A_{V U}(T),\left\{A_{U} ; U \in \mathbb{Q}_{u}\right\}$ is a semigroup. We have

$$
\left\|A_{U}(S)-A_{U}(T)\right\|_{p}=\left\|U^{*}(S-T) \cdot \bar{U}\right\|_{p}=\|S-T\|_{p}
$$

for $U \in \mathbb{Q}_{u}$ and $S, T \in L^{p}$. It follows from the Ryll-Nardzewski's fixed point theorem that there exists $H_{0} \in \mathcal{Q}$ such that $A_{U}\left(H_{0}\right)=H_{0}$ for all $U \in \mathbb{Q}_{u}$. This implies that $\overline{\delta(U)}=\bar{U} \cdot H_{0}-H_{0} \cdot \bar{U}$ for all $U \in \mathbb{Q}_{u}$. Since $\overline{Q_{b}}$ is a von Neumann algebra, each element of $\mathbb{Q}_{b}$ is a finite linear combination of elements of $\mathbb{Q}_{u}$. Thus we have $\overline{\delta(A)}=\left[\bar{A}, H_{0}\right]$ for all $A \in \mathbb{Q}_{b}$.

Now we set

$$
H_{p}=\frac{1}{2 i} \cdot\left(H_{0}-H_{0}^{*}\right) .
$$

Then we have $H_{p}=H_{p}^{*} \in L^{p}$ and $\overline{\delta(A)}=i \cdot\left[H_{p}, \bar{A}\right]$ for all $A \in Q_{b}$.

REMARK 4.2. In the same assumption as in Lemma 4.1, if $2 \lessgtr p<\infty$ we have $\delta(A) \xi=i\left(H_{p} A \xi-\bar{A} H_{p} \xi\right)$ for all $A \in \mathbb{Q}_{b}$ and $\xi \in L_{2}^{\omega}\left(\mathfrak{A}_{0}\right)$. In fact, we can easily show that $\xi \in \mathscr{D}\left(H_{p}\right)$ for each $\xi \in L_{2}^{\omega}\left(\mathfrak{U}_{0}\right)$. This implies that

$$
\delta(A) \xi=i \cdot\left[H_{p}, \bar{A}\right] \xi=i\left(H_{p} A \xi-\bar{A} H_{p} \xi\right)
$$

for all $A \in \mathbb{Q}_{b}$ and $\xi \in L_{2}^{\omega}\left(\mathfrak{A}_{0}\right)$.

LEMMA 4.3. Let $\mathfrak{A}$ be an unbounded Hilbert algebra over $\mathfrak{A}_{0}$ in $\mathfrak{B}$ and let $\mathbb{Q}$ be the left $E W^{\#}$-algebra of $\mathfrak{A}$. Then, for each $A$ in $Q$ there exists a sequence $\left\{B_{n}\right\}$ in $\mathbb{Q}_{b}$ which converges to $A$ with respect to $L^{p}$-norm for each $p(2 \leqslant p<\infty)$.

Proof. Take $A$ in $Q$ with $\bar{A}=T_{1}+T_{2}$ where $T_{1}$ is in $L^{\infty}$ and $T_{2} \in L_{2}^{\omega}\left(\phi_{0}\right)$. Let $T_{2}=U\left|T_{2}\right|$ be the polar decomposition and let $\left|T_{2}\right|=\int_{0}^{\infty} \lambda d E(\lambda)$ be the spectral resolution of $\left|T_{2}\right|$. We put

$$
B_{n}=T_{1}+U \int_{0}^{n} \lambda d E(\lambda), \quad n=1,2, \ldots,
$$

and, as easily seen, a sequence $\left\{B_{n}\right\}$ has the desired property.

Let $Q$ be a \#-algebra on $\mathscr{D}$ with the identity operator $I$ and let $\mathscr{G}$ be the completion of the pre-Hilbert space $\mathscr{D}$. We define the following seminorms on $\mathbb{Q}$ :

$$
\begin{gathered}
P_{\xi, \eta}(A)=|(A \xi \mid \eta)| \quad(\xi, \eta \in \mathscr{D}) ; \\
P_{\xi}(A)=\|A \xi\| \quad(\xi \in \mathscr{D}) .
\end{gathered}
$$

The locally convex topology on $Q$ generated by the family of the seminorms $\left\{P_{\xi, \eta}(\cdot) ; \xi, \eta \in \mathscr{D}\right\}$ (resp. $\left\{P_{\xi}(\cdot) ; \xi \in \mathscr{D}\right\}$ ) is called the weak (resp. strong) topology and is denoted by $t_{w}$ (resp. $t_{s}$ ).

THEOREM 4.4. Let $\mathfrak{A}$ be an unbounded Hilbert algebra over $\mathfrak{U}_{0}$ in $\mathfrak{Q}$ and let $\delta$ be a \#-derivation on the left $E W^{\#}$-algebra $\mathbb{Q}$ induced by $\mathfrak{A}$. If $\delta$ is $t_{s}$-continuous and the range of $\delta$ is contained in $\pi_{2}^{\omega}\left(L_{2}^{\omega}\left(\mathfrak{A}_{0}\right)\right)$, then for each $p(2 \leqslant p<\infty)$ there exists a selfadjoint operator $H_{p}$ in $L^{p}$ such that

$$
\delta(A) \xi=i \cdot\left[H_{p}, \bar{A}\right] \xi
$$

for all $A \in \mathbb{Q}$ and $\xi \in L_{2}^{\omega}\left(\mathfrak{A}_{0}\right)$. 
Proof. Take $p(2 \leqslant p<\infty)$. By Lemma 4.1, $\overline{\delta(B)}=i \cdot\left[H_{p}, \bar{B}\right]$ for all $B \in Q_{b}$. We show that $\overline{\delta(A)}=i \cdot\left[H_{p}, \bar{A}\right]$ for all $A \in \mathbb{Q}$. For each $A \in \mathbb{Q}$ there exists, by Lemma 4.3 , a sequence $\left\{B_{n}\right\}$ in $Q_{b}$ such that $\lim _{n \rightarrow \infty}\left\|\overline{B_{n}}-\bar{A}\right\|_{t}=0$ for all $t$ $(2 \leqslant t<\infty)$. Since $\delta$ is $t_{s}$-continuous, we have

$$
\lim _{n \rightarrow \infty}\left\|\left(\overline{\delta\left(B_{n}\right)}-\overline{\delta(A)}\right) \cdot \overline{\pi_{0}(\xi \eta)}\right\|_{1} \leqslant \lim _{n \rightarrow \infty}\left\|\delta\left(B_{n}\right) \xi-\delta(A) \xi\right\|\left\|\overline{\pi_{0}(\eta)}\right\|_{2}=0
$$

for every $\xi, \eta \in \mathfrak{A}_{0}$.

On the other hand, we have

$$
\begin{gathered}
\lim _{n \rightarrow \infty}\left\|H_{p} \cdot \overline{B_{n}} \cdot \overline{\pi_{0}(\xi \eta)}-H_{p} \cdot \bar{A} \cdot \overline{\pi_{0}(\xi \eta)}\right\|_{1} \leqslant \lim _{n \rightarrow \infty}\left\{\left\|H_{p}\right\|_{p}\left\|\left(\overline{B_{n}}-\bar{A}\right) \cdot \overline{\pi_{0}(\xi \eta)}\right\|_{p^{\prime}}\right\} \\
\leqslant \lim _{n \rightarrow \infty}\left\{\left\|H_{p}\right\|_{p}\left\|\overline{B_{n}}-\bar{A}\right\|_{q_{1}}\left\|\overline{\pi_{0}(\xi \eta)}\right\|_{q_{2}}\right\}=0
\end{gathered}
$$

where $1 / p+1 / p^{\prime}=1,1 / q_{1}+1 / q_{2}=1 / p^{\prime}$ and $q_{1}>2$, similarly

$$
\lim _{n \rightarrow \infty}\left\|\overline{B_{n}} \cdot H_{p} \cdot \overline{\pi_{0}(\xi \eta)}-\bar{A} \cdot H_{p} \cdot \overline{\pi_{0}(\xi \eta)}\right\|_{1}=0 .
$$

Therefore we have

$$
\begin{aligned}
\overline{\delta\left(B_{n}\right)} \cdot \overline{\pi_{0}(\xi \eta)} & =i\left(H_{p} \cdot \overline{B_{n}}-\overline{B_{n}} \cdot H_{p}\right) \cdot \overline{\pi_{0}(\xi \eta)} \\
& \rightarrow i\left(H_{p} \cdot \bar{A}-\bar{A} \cdot H_{p}\right) \cdot \overline{\pi_{0}(\xi \eta)}
\end{aligned}
$$

as $n \rightarrow \infty$ in $L^{1}$, which implies that

$$
\overline{\delta(A)} \cdot \overline{\pi_{0}(\xi \eta)}=i \cdot\left(H_{p} \cdot \bar{A}-\bar{A} \cdot H_{p}\right) \cdot \overline{\pi_{0}(\xi \eta)} .
$$

Since $\pi_{0}(\xi \eta)^{*}$ is a bounded operator on $\mathfrak{B}$, we have

$$
\begin{aligned}
\pi_{0}(\xi \eta)^{*} \delta(A)^{*} & =\pi_{0}(\xi \eta)^{*} \cdot \delta(A)^{*}=\left(\overline{\delta(A)} \cdot \overline{\pi_{0}(\xi \eta)}\right)^{*} \\
& =\pi_{0}(\xi \eta)^{*} \cdot\left(i \cdot\left[H_{p}, \bar{A}\right]\right)^{*}=\pi_{0}(\xi \eta)^{*}\left(i\left[H_{p}, A\right]\right)^{*},
\end{aligned}
$$

for every $\xi, \eta \in \mathfrak{A}_{0}$. Thus we have $\delta(A)^{*}=\left(i\left[H_{p}, \bar{A}\right]\right)^{*}$, which implies that $\overline{\delta(A)}=i$ - $\left[H_{p}, \bar{A}\right]$. This completes the proof of the theorem.

Using Remark 4.2 we can prove the following corollary by the similar calculations in the proof of Theorem 4.4.

COROllary 4.5. If $\delta$ is a $t_{w}$-continuous \#-derivation on the left $E W^{\#}$-algebra $\mathbb{Q}$ induced by $\mathfrak{A}$ with the range of $\delta$ in $\pi_{2}^{\omega}\left(L_{2}^{\omega}\left(\mathfrak{A}_{0}\right)\right)$, then for each $p(2<p<\infty)$ there exists a selfadjoint operator $H_{p}$ in $L^{p}$ such that

$$
\delta(A) \xi=i\left(H_{p} A \xi-\bar{A} H_{p} \xi\right)
$$

for all $A \in \mathbb{Q}$ and $\xi \in L_{2}^{\omega}\left(\mathfrak{A}_{0}\right)$.

From Theorem 4.4 and Corollary 4.5 we have the following

COROLlaRY 4.6. Let $\mathfrak{A}$ be an unbounded Hilbert algebra over $\mathfrak{A}_{0}$ in $\mathfrak{B}$ with identity and let $\delta$ be $a$ \#-derivation on the left $E W^{\#}$-algebra $\mathbb{Q}$ induced by $\mathfrak{A}$. If $\delta$ is $t_{s}$-continuous (or $t_{w}$-continuous), then for each $p(1<p<\infty)$ there exists $a$ selfadjoint operator $H_{p}$ in $L^{p}$ such that $\overline{\delta(A)}=i \cdot\left[H_{p}, \bar{A}\right]$ for all $A \in \mathbb{Q}$.

We recall the $\lambda$-topology defined by $D$. Arnal and J. P. Jurzak [1]. Let $\mathcal{Q}$ be a \#-algebra on $\mathscr{D}$ with the identity operator. For each $T \in \mathbb{Q}$ we set 


$$
\|A\|_{T}=\sup _{\xi \in \mathscr{D}} \frac{\|A \xi\|}{\|T \xi\|}, \quad A \in \mathbb{Q},
$$

where $\lambda / 0=\infty$ for $\lambda>0$. This defines the normed space $\mathfrak{N}_{T}=\left\{A \in \mathbb{Q} ; \lambda_{T}(A)\right.$ $<\infty\}$ equipped with the norm $\|\cdot\|_{T}$ and the spaces $\left\{\mathscr{R}_{T} ; T \in \mathbb{Q}\right\}$ constitute a direct set with $\cup_{T \in \mathscr{Q}} \mathfrak{K}_{T}=\Sigma_{T \in \mathscr{Q}} \mathfrak{N}_{T}=\mathscr{Q}$. The inductive limit topology for the normed spaces $\left\{\mathfrak{T}_{T} ; T \in \mathbb{Q}\right\}$ is called the $\lambda$-topology and is denoted by $t_{\lambda}$.

LEMMA 4.7. If $\mathbb{Q}$ is an $E W^{\#}$-algebra on $\mathscr{D}$, then for each $A$ in $\mathbb{Q}$ there exists a sequence $\left\{C_{n}\right\}$ in $\mathbb{Q}_{b}$ which converges to $A$ with respect to the $\lambda$-topology. Thus $\mathbb{Q}_{b}$ is dense in $\mathbb{Q}$ with respect to the $\lambda$-topology.

Proof. Take $A$ in $Q$. Let $\bar{A}=U|\bar{A}|$ be the polar decomposition of $\bar{A}$ and let $|\bar{A}|=\int_{0}^{\infty} \lambda d E(\lambda)$ be the spectral resolution of $|\bar{A}|$. Now we put $C_{n}=A E(n)$, $R_{n}=\int_{n}^{\infty}(1 / \lambda) d E(\lambda), n=1,2, \ldots$ Thus we have $C_{n} \in \mathbb{Q}_{b}$ and $\bar{A}-\overline{C_{n}}=\bar{A} R_{n}|\bar{A}|$. Furthermore, since $R_{n}$ commutes with $|\bar{A}|$, we have for each $\xi \in L_{2}^{\omega}\left(\mathfrak{A}_{0}\right)$

$$
\begin{aligned}
\left\|A \xi-C_{n} \xi\right\| & =\left\|\bar{A} R_{n}|\bar{A}| \xi\right\| \leqslant\left\||\bar{A}|^{2} R_{n} \xi\right\|=\left(\left.R_{n}^{2}|\bar{A}|^{2} \xi|| \bar{A}\right|^{2} \xi\right)^{1 / 2} \\
& \leqslant \frac{1}{n}\left(\left.(I-E(n))|\bar{A}|^{2} \xi|| \bar{A}\right|^{2} \xi\right)^{1 / 2} \leqslant \frac{1}{n}\left\||A|^{2} \xi\right\| .
\end{aligned}
$$

Hence we have $\left\|A-C_{n}\right\|_{A^{*} A} \leqslant 1 / n$. This implies that the sequence $\left\{C_{n}\right\}$ converges to $A$ with respect to the $\lambda$-topology.

Let $\mathcal{Q}$ be a \#-algebra on $\mathscr{D}$. We put $\tilde{\mathcal{D}}=\cap_{A \in \mathbb{Q}} \mathscr{D}(\bar{A}), \tilde{A} \xi=\bar{A} \xi$ for $A \in \mathbb{Q}$ and $\xi \in \tilde{\mathscr{D}}$. Then, $\tilde{\mathbb{Q}} \equiv\{\tilde{A} ; A \in \mathbb{Q}\}$ is a \#-algebra on $\tilde{D}$, which is called the closure of $\mathcal{Q}$. Let $\delta$ be a \#-derivation on $\mathcal{Q}$. We put $\tilde{\delta}(\tilde{A})=\tilde{\delta(A)}, A \in \mathbb{Q}$. Then $\tilde{\delta}$ is a \#-derivation on $\tilde{\mathbb{Q}}$.

THEOREM 4.8. Let $\mathfrak{A}$ be an unbounded Hilbert algebra over $\mathfrak{U}_{0}$ in $\mathfrak{B}$ and let $\delta$ be a \#-derivation on the left $E W^{\#}$-algebra induced by $\mathfrak{A}$ with the range of $\delta$ in $\pi_{2}^{\omega}\left(L_{2}^{\omega}\left(\mathfrak{A}_{0}\right)\right)$. If $\tilde{\delta}$ is continuous with respect to the topology $t_{w}\left(\right.$ or $t_{q w}, t_{\text {ow }}^{\mathbb{Q}}, t_{q \sigma w}^{\mathbb{Q}}, t_{\text {ow }}^{\mathscr{D}}, t_{\text {qow }}^{\mathbb{D}}$, $\left.t_{s}, t_{\sigma s}^{\mathbb{Q}}, t_{o s}^{\mathscr{D}}, t_{u}, t_{q u}, t_{\rho}, t_{\lambda}\right)$, then for each $p(2<p<\infty)$ there exists a selfadjoint operator $H_{p}$ in $L^{p}$ such that

$$
\delta(A) \xi=i\left(H_{p} A \xi-\tilde{A} H_{p} \xi\right)
$$

for all $A \in \mathbb{Q}$ and $\xi \in L_{2}^{\omega}\left(\mathfrak{A}_{0}\right)$. (Remark: the reader is referred to [1], [11] (resp. [13], [1]) for the topologies $t_{w}, t_{q w}, t_{\sigma w}^{\mathcal{Q}}, t_{q \sigma w}^{\mathbb{Q}}, t_{\sigma w}^{\mathbb{Q}}, t_{q o w}^{\mathbb{D}}, t_{s}, t_{\sigma s}^{\mathbb{Q}}$ and $t_{\sigma s}^{\mathscr{D}}\left(\right.$ resp. $t_{u}$ and $t_{q u}, t_{\rho}$ and $\left.t_{\lambda}\right)$.)

Proof. We first note from Remark 4.2 that $\delta(B) \xi=i\left(H_{p} B \xi-\bar{B} H_{p} \xi\right)$ for all $B \in \mathbb{Q}_{b}$ and $\xi \in L_{2}^{\omega}\left(\mathfrak{A}_{0}\right)$. From Lemma 4.7 , for each $A \in \mathbb{Q}$ there exists a sequence $\left\{C_{n}\right\}$ in $\mathbb{Q}_{b}$ such that $\left\{\tilde{C}_{n}\right\}$ converges to $\tilde{A}$ with respect to the $\lambda$-topology $t_{\lambda}$. Suppose $\tau$ is one of the following topologies: $t_{w}, t_{q w}, t_{\sigma w}^{\mathbb{Q}}, t_{q o w}^{\mathbb{Q}}, t_{\sigma w}^{\mathbb{D}}, t_{q o w}^{\mathbb{D}}, t_{s}, t_{o s}^{\mathbb{Q}}, t_{\sigma s}^{\mathbb{D}}, t_{u}$, $t_{q u}, t_{\rho}$ and $t_{\lambda}$. Since $t_{w} \leqslant \tau \leqslant t_{\lambda}\left[11\right.$, Theorem 2.1], it follows that $\left\{C_{n}\right\}$ converges to $\tilde{A}$ with respect to $\tau$. From the continuity of $\tilde{\delta}$ we have

$$
\lim _{n \rightarrow \infty}\left(\delta\left(C_{n}\right) \xi \mid \eta\right)=(\delta(A) \xi \mid \eta)
$$


for all $\xi, \eta \in L_{2}^{\omega}\left(\mathfrak{A}_{0}\right)$. Furthermore, it is easily shown that

$$
H_{p} \xi \in \widetilde{L_{2}^{\omega}\left(\mathfrak{A}_{0}\right)}
$$

for all $\xi \in L_{2}^{\omega}\left(\mathfrak{A}_{0}\right)$. This implies that

$$
\lim _{n \rightarrow \infty}\left(i\left(H_{p} C_{n}-C_{n} H_{p}\right) \xi \mid \eta\right)=\left(i\left(H_{p} A-\tilde{A} H_{p}\right) \xi \mid \eta\right)
$$

for all $\xi, \eta \in L_{2}^{\omega}\left(\mathfrak{A}_{0}\right)$. Hence we have $\delta(A) \xi=i\left(H_{p} A \xi-\tilde{A} H_{p} \xi\right)$ for all $A \in \mathbb{Q}$ and $\xi \in L_{2}^{\omega}\left(\mathfrak{A}_{0}\right)$. This completes the proof.

REMARK 4.9. Let $\mathfrak{A}$ be an unbounded left Hilbert algebra over $\mathfrak{U}_{0}$ in $\mathfrak{S}$ and let $\delta$ be a \#-derivation on the left $E W^{\#}$-algebra $\mathcal{Q}$ induced by $\mathfrak{A}$ with the range of $\delta$ in $\pi_{2}^{\omega}\left(L_{2}^{\omega}\left(\mathfrak{A}_{0}\right)\right)$. If $\delta$ is continuous with respect to the special topology (called ${ }_{\infty} L_{2}^{\omega}$ topology [10]) on $Q$, then the statement in Theorem 4.8 holds.

\section{REFERENCES}

1. D. Arnal and J. P. Jurzak, Topological aspects of algebras of unbounded operators, J. Funct. Anal. 24 (1977), 397-425.

2. O. Bratteli and D. W. Robinson, Unbounded derivations of $C^{*}$-algebras, Comm. Math. Phys. 42 (1975), 253-268.

3. __ Unbounded derivations of $C^{*}$-algebras. II, Comm. Math. Phys. 46 (1976), 11-30.

4. U Unbounded derivations of von Neumann algebras, Ann. Inst. H. Poincaré Sect. A 25 (1976), $139-164$.

5. O. Bratteli, Unbounded derivations of operator algebras, (CNRS Colloquium on Algebras of Operators and their Applications to Mathematical Physics, Marseille, 25-29 June 1977).

6. J. Dixmier, Les algèbres d'opérateurs dans l'espace Hilbertian, $2^{\dot{\epsilon}}$ ed., Gauthier-Villars, Paris, 1969.

7. P. R. Chernoff, Representations, automorphisms, and derivations of some operator algebras, J. Funct. Anal. 12 (1973), 275-289.

8. P. G. Dixon, Unbounded operator algebras, Proc. London Math. Soc. 23 (1971), 53-69.

9. A. Inoue, On a class of unbounded operator algebras. II, Pacific J. Math. 66 (1976), 411-431.

10. , Unbounded generalizations of standard von Neumann algebras, Rep. Math. Phys. 13 (1978), 25-35.

11. A. Inoue, K. Kuriyama and S. Ôta, Topologies on unbounded operator algebras, Mem. Fac. Sci. Kyushu Univ. Ser A 33 (1979), 355-375.

12. P. Kröger, Derivationen in $L^{+}(D)$, Beiträge zur Theorie nichtnormierbarer topologischer Algebren.

Wiss. 2, Karl Marx Univ. Leipzig Math.-Natur. Reihe 24 (1975), No. 5, 525-528.

13. G. Lassner, Topological algebras of operators, Rep. Math. Phys. 3 (1972), 279-293.

14. I. Namioka and E. Asplund, A geometric proof of Ryll-Nardzewski's fixed point theorem, Bull. Amer. Math. Soc. 73 (1967), 443-445.

15. R. T. Powers, Self-adjoint algebras of unbounded operators, Comm. Math. Phys. 21 (1971), 85-124.

16. J. R. Ringrose, Automatic continuity of derivations of operator algebras, J. London Math. Soc. 5 (1972), 432-438.

17. C. Ryll-Nardzewski, On fixed points of semigroups of endomorphisms of linear spaces, Proc. Fifth Berkeley Sympos. Math. Statist and Probability (Berkeley, Calif., 1965-1966), vol. II: Contributions to Probability Theory, Part I, Univ. California Press, Berkeley, Calif., 1967, pp. 55-61.

18. S. Sakai, Derivations of $W^{*}$-algebras, Ann. of Math. (2) 83 (1966), 273-279.

19. $C^{*}$-algebras and $W^{*}$-algebras, Springer-Verlag, Berlin-Heidelberg-New York, 1971.

20. __ Recent developments in the theory of unbounded derivations in $C^{*}$-algebras, (Proceeding, Second Japan-USA Seminar, Los Angeles, April 18-22, 1977), Lecture Notes in Math., vol. 650, Springer-Verlag, Berlin and New York, 1978.

21. I. E. Segal, A noncommutative extension of abstract integration, Ann. of Math. (2) 57 (1953), 401-457.

Department of APPlied Mathematics, Fukuoka University, Fukuoka 814, JaPAN

Department of Mathematics, Kyushu University 33, Fukuoka 812, Japan 